\title{
Conserved and newly acquired roles of PIF1 homologs in tomato (Solanum lycopersicum)
}

Miguel SIMON-MOYA ${ }^{1,2}$, M Victoria BARJA $^{2}$, Luca MORELLI ${ }^{1,2}$, Daniele ROSADO ${ }^{3}$, Linlin QI, Gianfranco DIRETTO ${ }^{4}$, Tomás MATUS ${ }^{5}$, Briardo LLORENTE,7, Jaime F. MARTINEZ-

GARCIA $^{1,2}$, Alain GOOSSENS ${ }^{8,9}$, Magdalena ROSSI ${ }^{3}$, Manuel RODRIGUEZ-CONCEPCION ${ }^{1,2, *}$

1, Institute for Plant Molecular and Cell Biology (IBMCP), CSIC-Universitat Politècnica de València, 46022 Valencia, Spain.

2, Center for Research in Agricultural Genomics (CRAG), CSIC-IRTA-UAB-UB, 08193 Barcelona, Spain

3, Departamento de Botânica, Instituto de Biociências, Universidade de São Paulo, , 05508-090 São Paulo, Brazil

4, Casaccia Research Centre, Italian National Agency for New Technologies, Energy, and Sustainable Development, 00123 Rome, Italy

5, Institute for Integrative Systems Biology (I2SysBio), Universitat de Valencia-CSIC, 46908 Paterna, Valencia, Spain

6, ARC Center of Excellence in Synthetic Biology, Department of Molecular Sciences, Macquarie University, NSW 2109 Sydney, Australia

7, CSIRO Synthetic Biology Future Science Platform, NSW 2109 Sydney, Australia

8, Department of Plant Biotechnology and Bioinformatics, Ghent University, 9052 Ghent, Belgium

9, VIB Center for Plant Systems Biology, 9052 Ghent, Belgium

* Corresponding author: Manuel RODRIGUEZ-CONCEPCION

Email: manuelrc@ibmcp.upv.es

ORCID number: 0000-0002-1280-2305 


\section{ABSTRACT}

PHYTOCHROME INTERACTING FACTORs (PIFs) are transcription factors that interact with the photoreceptors phytochromes and integrate multiple signaling pathways related to light, temperature, defense and hormone responses. PIFs have been extensively studied in Arabidopsis thaliana, but less is known about their roles in other species. Here, we investigate the role of the two homologs of PIF1 found in tomato (Solanum lycopersicum), namely PIF1a and PIF1b. Analysis of gene expression showed very different patterns, indicating a potential evolutionary divergence in their roles. At the protein level, light regulated the stability of PIF1a, but not PIF1b, further supporting a functional divergence. Phenotypic analyses of CRISPRCas9-generated tomato mutants defective in PIF1a or PIF1b or both revealed conserved and newly acquired roles compared to Arabidopsis PIF1. Both PIF1a or PIF1b were found to regulate seed germination, photosynthetic pigment biosynthesis and fruit production. However, only PIF1a-defective mutants showed defects on root hair elongation, flowering time and fruit growth and softening. We did not identify any process altered only in plants lacking PIF1b. Together, these data show that neofunctionalization has taken place in tomato, illustrating the potential of these transcription factors to acquiring new roles in different species. 


\section{INTRODUCTION}

Light is the main source of energy for plants but also a key source of environmental information that strongly influences plant developmental processes such as seed germination, phototropism, seedling development, chloroplast biogenesis and floral induction, among others. Plants are equipped with photoreceptors that detect and transduce light signals to trigger such responses. Phytochromes (phys) are probably one of the best known photoreceptors in plants, with five members (phyA to phyE) found in the model plant Arabidopsis thaliana. They are specialized in detecting and transducing light in the red (R) and far-red (FR) wavelengths. Phytochromes exist in two photoconvertible forms, an inactive $\mathrm{R}$ light-absorbing Pr form and an active FR light-absorbing Pfr form. The inactive Pr form is located in the citosol. Following the R light-mediated conversion, the Pfr form translocates to the nucleus where it can interact with a family of transcription factors called Phytochrome-Interacting Factors (PIFs). This interaction causes PIF phosphorylation and inactivation, eventually triggering a rapid and global transcriptional reprogramming (Park et al., 2012; Qiu et al., 2017; Park et al., 2018).

PIFs are members of the basic helix-loop-helix (bHLH) transcription factor superfamily (Ni et al., 1998). The Arabidopsis PIF family is composed of 8 members. All members have an Active phyB Binding domain (APB) but only PIF1 and PIF3 present an additional Active phyA Binding domain (APA) in their sequence (Leivar and Quail, 2011). Arabidopsis PIFs have been proposed as central integrators of light and other signaling pathways related to environmental (e.g. temperature and defense) and endogenous (e.g., hormone) cues (REF). Besides their role as transcription factors directly regulating the expression of thousands of genes, PIFs also interact with different types of regulatory proteins to modulate plant responses. (Leivar and Quail, 2011). In particular, Arabidopsis PIF1 is a repressor of seed germination, seedling photomorphogenesis (including chloroplast development and photosynthetic pigment chlorophylls and carotenoids- accumulation) and flowering (Toledo-Ortiz et al., 2010, 2014; Bou-Torrent et al., 2015; Chenge-Espinosa et al., 2018; Huq et al., 2004; Oh et al., 2004, 2006, 2007; Wu et al., 2018). PIF1 also functions in leaves, repressing carotenoid gene expression to adjust the levels of these important photoprotectors in response to environmental (i.e. light and temperature) signals (Toledo-Ortiz et al. 2010; Toledo-Ortiz et al. 2014). Similar to other PIFs, however, the role of PIF1 in other plant species remains much less studied.

In tomato (Solanum lycopersicum), two homologs of PIF1 have been reported, namely PIF1a (Solyc09g063010) and PIF1b (Solyc06g008030) (Llorente et al., 2016b, Rosado et al., 2016). PIF1a was proposed to repress carotenoid biosynthesis in green fruit, when the presence of chlorophylls prevents most $\mathrm{R}$ light to enter the fruit and hence causes a self-shade effect that promotes the accumulation of PIFs (Llorente et al., 2016a, 2016b). During ripening, chlorophylls degrade, resulting in lower PIF levels and derepressed production of carotenoid pigments that provide the red color of ripe fruit. Strikingly, PIF1a expression did not decrease but increased during ripening, a mechanism that was interpreted as a 'gas-and-brake' effect providing a more robust control of fruit carotenoid biosynthesis (Llorente et al., 2016a, 2016b).

Consistent with the negative role on the regulation of carotenoid biosynthesis reported for the members of the so called PIF quartet (PIFQ: PIF1, PIF3, PIF4 and PIF5) in Arabidopsis (Toledo-Ortiz et al., 2010, 2014; Bou-Torrent et al., 2015), silencing of the genes encoding PIF1a or PIF4 in tomato resulted in higher carotenoid levels (Llorente et al., 2016; Rosado et al., 2019). PIF4 was also found to be a regulator of thermomorphogenesis and inductor of leaf senescence and flowering in both Arabidopsis (Sakuraba et al., 2014; Song et al., 2014; Zhang et al., 2015) and tomato (Rosado et al., 2019). On the other hand, tomato PIF3 was identified as a regulator of steroidal glycoalkaloid (SGA) biosynthesis, a cholesterol-derived family of compounds that are not present in Arabidopsis but and contribute to pathogen defense in Solanaceae (Wang et al., 2018). Together, these results suggest that both evolutionary conservation and neofunctionalization occurs within the PIF family when comparing Arabidopsis and tomato. Here we aimed to further explore this possibility by focusing on PIF1 homologs. 


\section{RESULTS}

\section{Tomato PIF1 homologs show different expression profiles}

As an initial step to investigate whether the two PIF1 homologs present in tomato might play different physiological roles, we analyzed the expression pattern of both PIF1a and PIF1b genes using the Bio-Analytic Resource for Plant Biology (BAR, University of Toronto). According to these data (Fig. 1A), both PIF1a and PIF1b are expressed at similar levels in two organs: roots (where they both are low expressed) and leaves (where their transcript levels are much higher). Levels of PIF1a transcripts are higher than those of PIF1b in all stages of flower and fruit development. PIF1b expression increases as fruit grows until it reaches its final size at the mature green (MG) stage. During ripening, PIF1b expression does not change but PIF1a expression exhibits a strong increase as fruits move from the breaker (BR) to the red ripe (RR) stage. This result is in accordance with previously reported experimental data (Llorente et al., 2016b; Rosado et al., 2016). We next analyzed the gene co-expression networks (GCN) of PIF1a and PIF1b as an additional way of testing whether these two transcription factors might be involved in similar processes. We used data from TomExpress (Abdullah et al., 2017) to get a list of the 500 most highly co-expressed genes with PIF1a and PIF1b in three different organs: root, leaf and fruit (Supp Fig 1). Strikingly, we found virtually no overlapping of the GCNs of PIF1a and PIF1b in roots and leaves, whereas only about $10 \%$ of genes showed either positive (40 genes) or negative (62 genes) coexpression with both PIF1a and PIF1b in fruit (Supp Fig 1).

\section{A mutation in the APB domain of PIF1b impacts ligh-dependent stability but does not prevent heterodimerization with PIF1a}

Beyond expression patterns, protein stability is a key factor in the regulation of PIF activity. PIF1a and PIF1b are very similar in their amino acid sequence (primary structure), showing characteristic basic Helix-Loop-Helix (bHLH) and active phyB-binding (APB) domains like all PIFs plus an active phyA-binding (APA) domain that in Arabidopsis is only present in PIF1 and PIF3. However, the APB-binding domain of PIF1b presents an amino acid substitution that changes a conserved $Q$ residue to $G$ (Rosado et al., 2016). It was proposed that this sequence change could lead to a disrupted interaction with phyB and hence a differential light-dependent degradation compared to PIF1a (Bu et al., 2011b; Park et al., 2012). In order to experimentally confirm whether PIF1a and PIF1b abundance is differentially controlled by light, we used C-terminal GFP-tagged versions of these proteins transiently expressed in Nicotiana benthamiana leaves. Both PIF1a-GFP and PIF1b-GFP proteins were localized in nuclear bodies (Fig. 1B) (Gramegna et al., 2018), as expected for PIF transcription factors (Al-sady et al., 2006; Shen et al., 2005; Trupkin et al., 2014). Once we confirmed that the GFP-tagged proteins were correctly localized, we irradiated the agroinfiltrated leaf with either $\mathrm{R}$ or FR light for $30 \mathrm{~min}$ and took pictures of the same area before and after irradiation (Fig. $1 \mathrm{C}$ and Supp Fig 2). PIF1a-GFP fluorescence signal decreased after the exposure to $\mathrm{R}$ and increased after FR, a dynamic behavior that is typical of a PIF which interacts with phyB after a prolonged illumination (Shen et al., 2008). In contrast, PIF1b-GFP abundance was not altered by R or FR exposure (Fig. 1C and Supp Fig 2). It is therefore likely that, as predicted, the single amino acid change present in the APB domain of PIF1b prevents normal interaction with active phyB and hence disrupts its light regulation, likely impacting the biological activity of the protein.

Although it is not predicted that the mutation in the APB domain could interfere with the interaction of PIF1b with other proteins besides phyB, we decided to test it by taking advantage of the reported capacity of PIFs to interact with each other forming homodimers and heterodimers (Toledo-ortiz et al., 2003; Bu et al., 2011a). Using Bimolecular Fluorescence Complementation (BiFC), we confirmed that PIF1a and PIF1b are able to interact in the nucleus, more specifically in nuclear bodies, forming homodimers but also PIF1a-PIF1b heterodimers (Fig. 1D and Supp Fig 3). 
bioRxiv preprint doi: https://doi.org/10.1101/2021.10.29.466498; this version posted November 1 , 2021. The copyright holder for this preprint (which was not certified by peer review) is the author/funder, who has granted bioRxiv a license to display the preprint in perpetuity. It is made available under aCC-BY-NC-ND 4.0 International license.

\section{Single and double mutants were obtained using a CRISPR/Cas9 strategy}

In order to unveil the processes in which PIF1 homologs were involved, we generated knock-out lines in the tomato MicroTom variety using the CRISPR/Cas9 technology. We selected lines with a single nucleotide insertion for PIF1a and a two-nucleotide deletion for PIF1b. Both mutations caused a frameshift predicted to result in shorter PIF1a and PIF1b proteins that lacked the APA and bHLH domains, likely resulting in non-functional mutants (Supp Fig 4). We also generated double mutants and carried out multiple serial crosses with untransformed wild type (WT) plants to remove the CRISPR/Cas9 transgene and possible off-targets. We next used the resulting lines (pif1a, pif1b and pif1a pif1b) to test whether the loss of function of PIF1a, PIF1b, or both, interfered with the expression of other genes encoding PIFQ homologs (Supp Fig 5). The qPCR results showed no significant differences in transcript levels between WT and mutant leaves and MG fruit, with the only exception of decreased PIF1a transcript levels in PIF1a-defective mutants (Supp Fig 5).

\section{Both PIF1a and PIF1b repress germination under FR light.}

Arabidopsis PIF1 is an essential player in light-regulated seed germination. Under FR light, PIF1 accumulates in the nucleus and represses the germination process, while in the presence of $\mathrm{R}$ light, the activated phys degrade PIF1 and germination takes place (Oh et al., 2004; Seo et al., 2009; Shinomura et al., 1994; Lee et al., 2012; Shi et al., 2013). Similar to Arabidopsis, germination of tomato seeds is repressed by FR light and promoted by $\mathrm{R}$ light (Shichijo et al., 2001; Appenroth et al., 2006; Auge et al., 2009). To test whether tomato PIF1 homologs might be involved in the light-dependent regulation of seed germination, we exposed seeds from WT and mutant lines to hourly pulses of R or FR light as previously described (Appenroth et al., 2006). Treatment with $\mathrm{R}$ light resulted in similar germination rates for WT and mutant pif1a, pif1b and pif1a pif1b seeds (Fig. 2A). By contrast, FR light treatment repressed germination of WT seeds, as expected, but had little impact on the germination of mutant seeds (Fig. 2A). This indicates that both PIF1a and PIF1b repress seed germination in tomato under FR light, similar to that observed for PIF1 in Arabidopsis.

\section{PIF1a and PIF1b do not repress but induce photosynthetic pigment production.}

Arabidopsis PIF1 was shown to negatively regulate carotenoid and chlorophyll biosynthesis during seedling de-etiolation (Huq et al., 2004; Moon et al., 2008; Toledo-Ortiz et al., 2010). PIF1 accumulates in dark-grown (i.e., etiolated) seedlings, directly repressing the expression of genes supporting the production of photosynthetic pigments and the differentiation of chloroplasts. In the dark-to-light transition, PIF1 is degraded, allowing rapid biosynthesis and accumulation of chlorophylls and carotenoids and the transformation of etioplasts into chloroplasts (Toledo-Ortiz et al., 2010, 2014; Bou-Torrent et al., 2015). To test whether tomato PIF1 homologues contributed to carotenoid and chlorophyll production during seedling de-etiolation, we germinated WT and mutant seeds in the dark for 7 days, and then illuminated them with white light for an additional day. Samples were collected at different times after illumination for measuring photosynthetic pigment levels by HPLC. Plants germinated and grown in the light were used as controls. In stark contrast with the results reported in Arabidopsis, the absence of PIF1a, PIF1b, or both, in tomato seedlings led to a deceleration in the light-triggered accumulation of carotenoids and chlorophylls (Fig. 2B). A similar phenotype has been reported in PIF-defective Arabidopsis mutants when they were left for too long in the dark (Monte et al., 2004). In the case of tomato, however, reduced levels of photosynthetic pigments were also detected in mutant seedlings that were germinated and growth for 7 days in the light (Fig. 2B), suggesting that the tomato PIF1 homologs might not be repressors but activators of chlorophyll and carotenoid biosynthesis in photosynthetic tissues. 
bioRxiv preprint doi: https://doi.org/10.1101/2021.10.29.466498; this version posted November $1,2021$. The copyright holder for this preprint (which was not certified by peer review) is the author/funder, who has granted bioRxiv a license to display the preprint in perpetuity. It is made available under aCC-BY-NC-ND 4.0 International license.

\section{Root hair elongation is blocked only in PIF1a-defective lines}

While doing the germination and de-etiolation experiments, we noticed that tomato single pif1a and double pif1a pif1b mutants had hairless roots (Fig. 3A), a phenotype that has never been reported in PIF-deficient Arabidopsis mutants. We confirmed that Arabidopsis pifq mutants defective in PIF1 but also in the other members of the PIF quartet showed no obvious defects in root hair development (Fig. 3B). Scanning Electron Microscope (SEM) analysis of tomato roots showed that root hair primordia were indeed present in pif1a and pif1a pif1b roots, suggesting that the loss of PIF1a activity does not interfere with root hair initiation but prevents elongation (Fig. 3C). This phenotype was very robust, affecting all the seedlings in homozygous populations of single pif1a and double pif1a pif $1 b$ mutants, and being absent from WT and pif1 $b$ mutants. Therefore, the results suggest that PIF1a, but not PIF1b, is required for root hair elongation in tomato.

\section{Loss of PIF1a might result in early flowering}

Arabidopsis pif1 mutants show early flowering and up-regulated expression of the major flowering-promoting genes (Wu et al., 2018). We assessed the flowering time of tomato pif1a and pif1b mutants by measuring two different parameters: (1) the number of leaves produced when the first flower reached anthesis and (2) the number of days from sowing to anthesis. The first parameter (number of leaves) indicated a slight but statistically significant reduction of flowering time (i.e. early flowering) in single pif1a and double pif1a pif1b mutants, but not in pif1b plants (Supp Fig 6). By contrast, the second parameter (number of days) showed no differences between genotypes (Supp Fig 6). A number of other studies in the literature report differences in flowering when using one of the evaluated parameters but not when using the other (Calvert, 1959; Giliberto et al., 2005).

\section{Fruits defective in PIF1 homologs show normal carotenoid and ethylene production}

Despite the obvious differences between tomato and Arabidopsis fruits, many of the genes known to be involved in the control of tomato fruit development and ripening are homologous to Arabidopsis genes controlling different developmental processes (Itkin et al., 2009; Gapper et al., 2013; Seymour et al., 2013; Pesaresi et al., 2014). In particular, genes identified to participate in light signaling in Arabidopsis were later found to be involved in fruit development and ripening in tomato (Monte et al., 2004; Bianchetti et al., 2018; Lupi et al., 2019). Using our tomato mutants defective in PIF1a and PIF1b, we aimed to investigate possible roles of these two homologs in different processes associated with tomato fruit development.

PIF1a was previously found to repress carotenoid biosynthesis during ripening (Llorente et al., 2016b). Fruits from transgenic lines with a partially silenced PIF1a gene were found to accumulate higher levels of carotenoids when ripe (Llorente et al., 2016b), and a similar phenotype has been recently reported in PIF4-silenced fruits (Rosado et al., 2019). The levels of carotenoids but also other related isoprenoid metabolites regulated by PIFs (chlorophylls and tocopherols) were quantified by HPLC in WT and mutant fruits at three different ripening stages, from MG to RR. Strikingly, virtually no differences were found in the levels of any of these metabolites between WT and mutant fruit in any of the stages analyzed (Supp Fig 7). Consistently, fruit pigmentation was normal in all mutant lines. Carotenoid production and many other processes associated with fruit ripening are regulated by the hormone ethylene (Giovannoni, 2004). Because an important crosstalk between PIFs and many plant hormones (including ethylene) has been described in Arabidopsis (Lau and Deng, 2010; de Lucas and Prat, 2014), we next tested whether ethylene metabolism was affected during fruit ripening of the tomato PIF1-defective mutant lines by GC-MS (Pereira et al., 2017). We found no significant differences between genotypes (Supp Fig 7), concluding that none of the tomato PIF1 homologs regulates ethylene production during fruit ripening. 


\section{Tomato fruit size, weight and texture are regulated by PIF1a but not PIF1b}

Down-regulation of PIF4 leads to a decrease in fruit yield and size (Rosado et al., 2019). In order to test whether the tomato PIF1 homologs might also participate in the regulation of fruit yield and size, we quantified the number and volume of fruits produced by mutant lines. Interestingly, pif1a mutant plants produced less fruits than WT controls, while no differences were found in the case of pif1b or pif1a pif1b lines (Fig. 4A). Fruit size was estimated by measuring the volume of groups of 10 fruits harvested from the plant at the RR stage. Opposite to that observed in the case of PIF4-silenced fruits (Rosado et al., 2019), pif1a and pif1a pif1b fruits were found to be bigger than WT or pif1b fruits (Fig. 4B). We next measured the weight of 100 individual RR fruits from each genotype and confirmed that single pif1a and double pif1a pif1b mutant plants developed not only bigger but also heavier fruits (Fig. 4C). Furthermore, we confirmed that the difference in weight in PIF1a-defective fruits was maintained after drying the fruits (Supp Fig 8), which means that it is not due to increased water content but results from an enhanced accumulation of dry matter.

The observation that pif1a mutants produced more and bigger fruits prompted us to investigate whether the texture of ripe PIF1a-deficient fruits was also altered. In order to measure fruit hardness, we used a texture analyzer fitted with $50 \mathrm{~mm}$ plate probe to perform a compression test (Kabas and Ozmerzi, 2008; Camps and Gilli, 2017). This test provides a texture parameter called Wc, which is the mechanical work needed to reach a $5 \%$ deformation of the fruit. Higher Wc values were obtained in the case of pif1a and pif1a pif1b mutants (Fig 4D), indicating a higher resistance to deformation (i.e., harder fruit).

\section{DISCUSSION}

\section{PIF1a and PIF1b work together to regulate seed germination, photosynthetic pigment biosynthesis and fruit production}

Several lines of evidence support the conclusion that duplicated copies of the PIF1 gene in tomato have functionally diverged. The first one is the differential protein stability of PIF1a and PIF1b under different light conditions. Our data suggest that the amino acid substitution in the APB-binding domain of PIF1b likely leads to a failure in the interaction with phyB (Fig. 1). This result suggests that during evolution, PIF1b may have lost its capacity to transduce phyB-mediated light signals. Contrasting with this, PIF1b-defective lines showed lightdependent phenotypes during seed germination (Fig. 2A) and seedling de-etiolation (Fig. $2 \mathrm{~B})$. It has been reported that phyB regulates the $R$ light-induction and $F R$ light-inhibition of seed germination when using single light pulses (Appenroth et al., 2006). In contrast, phyA would be involved in the induction and inhibition when the pulses were applied hourly (as we did in our experiment). Therefore, the lack of interaction with phyB would not be a problem to transduce the phyA-dependent light signal mediating the germination response under a multi-pulse experimental system. The involvement of phyA and other phys besides phyB in the control of photosynthetic pigment accumulation during seedling deetiolation has been also described (Su et al., 2015). In fact, phyA has been described as the photoreceptor with a major contribution to initiating photomorphogenesis in Arabidopsis (Casal et al., 2003; Seo et al., 2004). This predominant role of phyA would also explain why PIF1b might regulate this process despite being unable to interact with phyB.

Light-dependent seed germination and photosynthetic pigment biosynthesis during seedling de-etiolation are regulated by both PIF1a and PIF1b proteins (Fig. 5). In fact, loss of any of the two PIF1 homologs produces the same phenotype than the lack of both in double mutants (Fig. 2A and 2B). This made us think that both PIF1a and PIF1b are part of the same signal transduction pathway. We speculate that there might be a threshold amount of PIF1 activity needed to trigger the signaling pathways required to inhibit germination or to accumulate photosynthetic pigments during seedling development. In WT lines, both PIF1a 
and PIF1b are required to reach this threshold. If one of the genes is lost, the remaining amount of PIF1 activity would not be enough to normally trigger the process. Loss of all tomato PIF1 activity in double pif1a pif1b mutants would have the same effect observed in single mutants, i.e. when the threshold is not met by losing only one of the two genes. Another possible scenario based on the BiFC result (Fig. 1D) is that maybe PIF1a and PIF1b need to form a heterodimer to regulate the expression of the genes involved in seed germination and photosynthetic pigment accumulation during de-etiolation. So, when one of the elements of this complex is missing, these processes are affected (Fig. 5). This complex would be interacting with phyA through both of the elements or with phyB through just PIF1a.

Another conclusion based on the observed phenotypes is that PIF1 proteins may function as activators or repressors depending on the biological context. In Arabidopsis, the pif1 mutant accumulates higher levels of carotenoids in the dark and produces more carotenoids and chlorophylls during de-etiolation (Huq et al., 2004; Moon et al., 2008; Toledo-Ortiz et al., 2010). In contrast, our results with tomato PIF1-defective mutants show only slightly increased levels of carotenoids in dark-grown seedlings but decreased levels of photosynthetic pigments in de-etiolating and light-grown seedlings (Fig. 2B). These results suggest that tomato PIF1a and PIF1b homologs might not be repressors but activators of photosynthetic development and leaf pigment biosynthesis (Fig. 5). Analysis of PIF1adefective mutants of other Solanaceae species should provide valuable information on whether this is a general or species-specific phenotype.

Both PIF1a and PIF1b might also participate in the control of fruit production (i.e. total number of fruits per plant), as this parameter was reduced in single pif1a but not in double pif1a pif1b lines (Fig. 4A). We hypothesize that PIF1a-PIF1b heterodimers might activate fruit production whereas PIF1b-PIF1b homodimers might repress it (Fig. 5). The WT phenotype would result from a balance between activation and repression. Loss of PIF1a would lead to only repression (by PIF1b-PIF1b homodimers), whereas additional loss of PIF1b would remove both activation and repression pathways, resulting in a newly balanced situation and hence a WT phenotype in pif1b and pif1a pif1b plants. While other scenarios are possible, antagonistic roles of light signaling homologs are common. In Arabidopsis, PIF2 and PIF6 have antagonistic roles with PIF7 and PIFQ proteins for the control of lighttriggered seedling de-etiolation (Pham et al., 2018). In the case of PIF2, it positively regulates seedling de-etiolation and photomorphogenesis by interacting with PIF1 and other PIFQ members, preventing them from regulating their target genes (Pham et al., 2018).

\section{PIF1a is the main PIF1 homolog regulating many other processes, including some that are not regulated by PIFs in Arabidopsis}

Phenotypic analysis of the CRISPR/Cas9 mutants unveiled that there are many processes that would be regulated by just PIF1a. A striking case is the root hair elongation phenotype, which is not present in PIFQ-defective Arabidopsis mutants (Fig. 3). Arabidopsis phyB mutant lines were reported to develop longer root hairs (Reed et al., 1993), but it is possible that this phenotype depends on factors other than PIFs in Arabidopsis. Alternatively, scarcely explored PIFs (such as PIF2, PIF6 or PIF8) might have a major role in this process. Note that PIFs such as PIF4 and PIF5 have been identified as growth promoters in other organs, (Choi and Oh, 2016), notably hypocotyl elongation in response to shade (De Lucas et al., 2008), blue light (Pedmale et al., 2016) or temperature (Koini et al., 2009; Thines et al., 2014). Maybe during evolution, a neofunctionalization event took place and PIF1a acquired this elongation-promoting role specifically in root hairs (Fig. 5).

Flowering time also seems to be promoted by PIF1a, but not by PIF1b (Fig. 2D). Even though the differences between PIF1a-defective and WT lines are relatively small and are only observed when counting the number of leaves from germination to anthesis (but not when counting the number of days), they are statistically significant and consistent with the observation that PIF1 regulates flowering time in Arabidopsis (Wu et al., 2018) (Fig. 5). A 
bioRxiv preprint doi: https://doi.org/10.1101/2021.10.29.466498; this version posted November 1 , 2021. The copyright holder for this preprint (which was not certified by peer review) is the author/funder, who has granted bioRxiv a license to display the preprint in perpetuity. It is made available under aCC-BY-NC-ND 4.0 International license.

number of other studies in the literature report differences in flowering when using one of the evaluated parameters but not when using the other (Calvert, 1959; Giliberto et al., 2005). Note that we grew our plants in the greenhouse under long day photoperiod, while domesticated tomato, in contrast with Arabidopsis, has been described as a day-neutral species (Soyk et al., 2017). Arabidopsis PIF4 and PIF5 were reported as thermosensory regulators of flowering (Kumar et al., 2012; Thines et al., 2014). Despite this, tomato PIF4 seems not to be related with this process, since the down-regulation of its expression leads to a reduction in flowers per truss, but not in flowering time (Rosado et al., 2019).

While seed germination, seedling de-etiolation, root hair development and flowering are biological processes present in both Arabidopsis and tomato, there are tomato-specific processes that are also regulated by PIF1a, such as those associated with fruit ripening. PIF1a is the only PIF-encoding gene that is up-regulated during fruit ripening (Rosado et al., 2016). One of the most characteristic features of tomato fruit ripening is the degradation of chlorophylls and the massive production of carotenoids when MG fruits start to ripe, changing their green color to red in the RR stage. PIF1a was first identified as a repressor of fruit carotenoid synthesis during ripening (Llorente et al., 2016b). In that work, an artificial microRNA approach was used to down-regulate PIF1a gene expression about $75 \%$, resulting in ripe fruits that accumulated higher levels of total carotenoids (Llorente et al., 2016b). In contrast with those results, WT levels of carotenoids were detected in fruits from the pif1a mutant line generated in this work, which is predicted to be completely devoid of PIF1a (Supp Fig 7). An explanation for this (lack of) phenotype came from the careful analysis of off-target effects in the two studies that used gene knockdown approaches to conclude that PIF1a and PIF4 are repressors of carotenoid biosynthesis during fruit ripening (Llorente et al., 2016b; Rosado et al., 2019). In PIF1a-silenced fruits (Llorente et al., 2016b), the most strongly down-regulated off-targeted PIF was PIF4 ( 40\%), while in PIF4-silenced fruits (Rosado et al., 2019) the most strongly down-regulated off-targeted PIF was PIF1a ( 60\%). Taking all these data together, we propose two possible explanations. First, PIF1a and PIF4 might be functionally redundant and play a similar role in modulating fruit carotenoid biosynthesis during ripening. This hypothesis involves that higher carotenoid contents would only be observed when both PIF1a and PIF4 are down-regulated. Because PIF4 expression is not affected in the pif1a mutant generated in this work (Supp Fig 5), PIF4 levels would be high enough to repress carotenoid overaccumulation in the pif1a line. The second hypothesis states that PIF4, but not PIF1a, would be involved in repressing carotenoid production during ripening. Thus, reduced PIF4 transcript levels in fruits of knockdown PIF1a and PIF4 lines, but not in our CRISPR-Cas9 mutants, would lead to higher carotenoid contents.

Fruit growth in terms of size (Fig. 4B) and weight (Fig. 4C) increased in pif1a but also in the pif1a pif1b double mutant. Based on the results of the fruit desiccation experiment (Supp Fig. 8) we concluded that the difference in size might be due to a difference in tissue mass, not in water content of the fruits, pointing out PIF1a as a repressor of fruit growth (Fig. 5). Arabidopsis PIF1 has been shown to regulate the expression of cell-wall-related genes (Oh et al., 2009; Shi et al., 2013). Alteration of cell-wall structure by PIF1a might explain the fruit softening phenotype of PIF1a-defective fruits (Fig 4D). Thus, PIF1a might be involved in the loosening of the cell-wall to allow root hair elongation and fruit softening while repressing fruit growth by a different mechanism (Fig. 5). This contrasting and opposite role of PIF1a in different tissues and developmental stages can be due to different interactions with different partners depending on the tissue and the developmental stage. For instance, protein-protein interaction between PIFs and with other factors can modulate their capacity to bind to the DNA (Pham et al., 2018). In this way, PIF1a might have specific interactors to promote root hair elongation or ripe fruit softening, while a different set of interactors would repress fruit growth.

Arabidopsis PIF4 has an important role in promoting hypocotyl elongation in response to different light and temperature cues (Koini et al., 2009; Choi and Oh, 2016; Pham et al., 
2018). Supporting the conclusion that PIF4 has conserved a growth-promoting role in different plant species and tissues, tomato knock-down lines for PIF4 develop smaller fruits (Rosado et al., 2019). Therefore, PIF1a and PIF4 appear to play antagonistic roles in the regulation of fruit size. A closer look at the expression patterns of the genes encoding PIF1a and PIF4 during fruit development shows that the expression of PIF1a is low at the early stages of fruit development (when growth takes place by cell division and proliferation) and slowly increases as cells expand (until the MG stage) and fruits begin to ripe, peaking at the RR stage (Supp Fig 9). In contrast, PIF4 expression peaks at the MG stage (i.e. when fruit reaches its final size) and then drops during ripening (Supp Fig 8). Based on these data, we speculate that PIF4 is the main promoter of fruit growth. Similar to that discussed above on the opposite role of PIF1a-PIF1b heterodimers and PIF1b homodimers for the regulation of fruit production (Fig. 5), it is possible that PIF1a-PIF4 heterodimers could antagonize the activating role of PIF4, perhaps by preventing binding of PIF4 homodimers to fruit-promoting target genes. As PIF1a levels increase during fruit development, heterodimers become more abundant and PIF4 homodimers decrease, resulting in a progressive attenuation of growth. Then, after the MG stage, PIF4 levels drop and growth stops. The peak of PIF1a expression at the RR stage is most likely unrelated to growth but required for fruit softening. Whether tomato PIF4 also interacts with PIF1a to regulate root hair elongation remains unknown.

\section{Evolutionary implications of PIF1 duplication}

As a summary of this work, graphically represented in Fig. 5, we conclude that some of the PIF1-regulated processes in Arabidopsis are also regulated by PIF1 homologs in tomato (including seed germination, photosynthetic pigment accumulation and flowering time). A second group includes processes that are regulated by PIF1 homologs in tomato but not by PIF1 in Arabidopsis. From them, some occur in both plant species (such as root hair elongation) but others are specific of tomato (fruit ripening). Moreover, the duplication of PIF1 present in tomato led to some of the above-mentioned processes being regulated by both PIF1a and PIF1b (seed germination, photosynthetic pigment accumulation and fruit production) while the rest became controlled just by PIF1a. Interestingly, we did not find any process that would be regulated exclusively by PIF1b (Fig. 5).

The duplication of PIF1 has been analyzed in detail previously (Rosado et al., 2016). The duplication of PIF1 in PIF1a and PIF1b is predicted to have happened just before the divergence between tomato and potato (Rosado et al., 2016; Sato et al., 2012). We hypothesize that before the divergence between Arabidopsis and Solanum there were likely some processes that were already regulated by PIF1 homologs in both species (e.g., seed germination, photosynthetic pigment contents or flowering time). During the following millions of years, the duplication of PIF1 in tomato led to newly acquired functions. While PIF1a remained as the main isoform, the presence of PIF1b provided robustness to essential processes (e.g. seed germination) and allowed to regulate new processes via heterodimerization (e.g. fruit production). On the other hand, some pre-existing processes regulated by PIF1 in Arabidopsis became controlled just by PIF1a in tomato, such as flowering time. The reason behind this might have been the mutation identified in PIF1b that leads to the loss of interaction with phyB. PIF1a also acquired the capacity to regulate new processes. The root hair elongation phenotype is a striking example with two possible explanations. The first one is a neofunctionalization process, by which PIF1a ended up involved in the regulation of the elongation of this cell type. A second possibility is that the original PIF1 was controlling this trait, but during evolution this role was lost in Arabidopsis but remained in tomato. In fruit, PIF1a and, likely, PIF4 might have re-adapted their already existing role in the regulation of cell elongation (Paik et al., 2017) to determine the final fruit size. There are several examples of transcription factors that have been recycled during evolution to regulate new processes, like fruit ripening (Gapper et al., 2013). For instance, in Arabidopsis SHATTERPROOF 1 (SHP1) and SHP2 are important regulators of valve margin identity and its subsequent dehiscence zone (Liljegren et al., 2000). By contrast, their closest 
bioRxiv preprint doi: https://doi.org/10.1101/2021.10.29.466498; this version posted November 1 , 2021. The copyright holder for this preprint (which was not certified by peer review) is the author/funder, who has granted bioRxiv a license to display the preprint in perpetuity. It is made available under aCC-BY-NC-ND 4.0 International license.

homolog in tomato, TOMATO AGAMOUSLIKE1 (TAGL1) controls fleshy fruit expansion (Itkin et al., 2009; Vrebalov et al., 2009; Gimenez et al., 2010). Another example is SEPALLATA 4 (SEP4), which in Arabidopsis regulates organ identity in flowers (Ditta et al., 2004). The closest homolog to SEP4 in tomato in RIPENING INHIBITOR (RIN), which is a key regulator of fruit ripening and controls climacteric respiration and ethylene biosynthesis (Fujisawa et al., 2011; Martel et al., 2011). Our results suggest that processes of specialization and neofunctionalization besides those detected here by analyzing the phenotypes of CRISPR-Cas9 mutants would have taken place during evolution after the splitting of Arabidopsis and Solanum groups. Further approaches, such as transcriptomics or metabolomic analysis, would provide some clues about which new tomato processes became regulated by PIF1a and PIF1b.

\section{ACKNOWLEDGEMENTS}

We thank greatly Jordi Gine-Bordanaba for assistance with the texture analyzer and $\mathrm{M}$. Rosa Rodriguez-Goberna for technical help. The work was funded by Spanish grants BIO2017-84041-P and PID2020-115810GB-I00 from the Agencia Estatal de Investigacion (AEI) and FEDER and 202040E299 from Consejo Superior de Investigaciones Científicas (CSIC) to MRC. We also acknowledge the AEl support for the PRIMA project UToPIQ (PCl2021-121941) and the Generalitat Valenciana grant PROMETEU/2021/056. MSM was supported by PhD fellowship BES-2015-072725 from the Spanish Ministerio de Economia y Competitividad. M.V.B. was funded with a Spanish Ministry of Education, Culture and Sports PhD fellowship (FPU14/05142). L.M. received a predoctoral fellowships from La Caixa Foundation (INPhINIT fellowship LCF/BQ/IN18/11660004) which received funding from the EU-H2020 through MSCA Grant 713673.

\section{MATERIALS AND METHODS}

\section{Plant material and growth conditions}

Nicotiana benthamiana and tomato plants were grown under standard long day greenhouse conditions (14 $\mathrm{h}$ light at $27 \pm 1^{\circ} \mathrm{C}$ and $10 \mathrm{~h}$ dark at $24 \pm 1^{\circ} \mathrm{C}$ ). Tomato plants were transformed as previously described (Fernandez et al., 2009). Seed germination assays were performed as was previously described (Appenroth et al., 2006). Briefly, Tomato seeds were surface-sterilized for $10 \mathrm{~min}$ with $40 \%(\mathrm{v} / \mathrm{v}) \mathrm{NaClO}$, washed 3 times with sterile water and sown on sterile Murashige and Skoog (MS) medium containing $1 \%$ agar and no sucrose. After that, seeds were incubated in dark conditions and exposed hourly to 5-min $R$ $\left(200 \mu \mathrm{mol} \mathrm{m} \mathrm{s}^{-1}\right)$ or FR $\left(100 \mu \mathrm{mol} \mathrm{m} \mathrm{s}^{-2}\right)$. Temperature was always kept at $25^{\circ} \mathrm{C}$. Radicle protrusion was used as a criterion for judging seed germination. For de-etiolation experiments, tomato seeds were surface-sterilized for 10 min with $40 \%(\mathrm{v} / \mathrm{v}) \mathrm{NaClO}$, washed 3 times with sterile water and sown on sterile wet cotton. After that, seed were incubated in dark conditions, except the Light control of the experiment, that was incubated in continuous light conditions. After 7 days, the seedlings were transferred to light. Samples were collected at the indicated time points after exposure to light. Temperature was always kept at $25^{\circ} \mathrm{C}$. Flowering time was assessed as was previously reported in tomato (Dielen et al., 1998). Basically, after sowing the seeds we waited until the first flower reached the anthesis stage. In that moment we measure two parameters: The number of leaves and the days postsowing when the anthesis took place. Fruit production was measured by counting the number of produced fruits per plants in 19-weeks-old plants in greenhouse conditions. The volume of the fruits was assessed by measuring the displaced volume in a graduated cylinder of a group of 10 representative fruits. The weight of the fruits was assessed by two 
different methods. First, 100 fruits were weight individually. Second, 15 fruits were weight together as a group. This second method was used to compare the weight difference between fresh samples and dry samples. To dry the fruits, all the 15-fruits groups were incubated for 4 days at $90^{\circ} \mathrm{C}$. Fruit hardness was measured with a texture analyzer (Stable Micro Systems, TA-XT2i) fitted with $50 \mathrm{~mm}$ plate probe to perform a compression test (Kabas and Ozmerzi, 2008; Camps and Gilli, 2017). The instrument was set to measure the mechanical work needed to reach $5 \%$ deformation of the original form of the fruit.

\section{Transient expression assays}

Leaves from 4-week-old $N$. benthamiana plants were entirely infiltrated with the desired combination of Agrobatoerium tumefaciens strains in the infiltration buffer (10 mM MES pH5.5.-6, $10 \mathrm{mM} \mathrm{MgSO4}$, acetosyringone $100 \mu \mathrm{M})$. After agroinfiltration, the plants were left in the greenhouse for 3 days. Leaves were used to perform confocal microscopy analysis. In the case of BiFC experiments, equal volumes of Agrobacterium cultures were mixed to get the indicated combinations in Fig. 1D and Supp Fig 3.

\section{DNA constructs and genotyping}

The coding sequences of PIF1a and PIF1b were cloned into a gateway ENTRY vector (pDNOR207, Thermo Fisher). After that, using a gateway LR reaction (Thermo Fisher), the CDSs were transferred to the final expression vectors, in frame with the GFP gene for subcellular localization (pDONR221 and pK7FWG2), and with $\mathrm{N}$ - or C-terminal sequence of GFP for BiFC experiments (pYFN43 and pYFC43 respectively) (Belda-Palazón et al., 2012). For the CRISPR/Cas9 constructs two guide RNAs (sgRNA) were cloned via cut-ligation reaction with Bbsl (Thermo) and T4 DNA ligase (Thermo) in a Gateway ENTRY sgRNA shuttle vector (Ritter et al., 2017). Next, using a gateway LR reaction (Thermo Fisher), the two sgRNA modules were then combined with pDE-Cas9Km vector (Ritter et al., 2017) to yield the final expression clone. First identification of CRISPR/Cas9 mutations was assessed with the TIDE (Tracking of Indels by DEcomposition) web tool. We analyze the chromatograms individually to identify the short insertion or deletion, using as template a chromatogram of a WT plant. The alignment was performed in Benchling (www.benchling.com) using MAFFT as an alignment program. Once the insertions or deletions were identified, CRISPR/Cas9 lines were genotyped using a PCR to amplify 500 $\mathrm{bp}$ of the mutated version. During the identification of the mutation we detected that the mutation in PIF1a removed a restriction site for Drdl and the mutation in PIF1b removed a restriction site for NlalV. The amplified fragments were then digested with the indicated enzymes.

\section{Gene expression profiling}

Expression data of different tissues were retrieved from the Bio-Analytic Resource for Plant Biology (BAR, University of Toronto), using the tomato tool available in that website and PIF1a to PIF1b Solyc IDs. Co-expression analysis was performed as previously described (Ahrazem et al., 2018). Briefly, tomato PIF1a to PIF1b Solyc IDs were used to retrieve all the expression data available for different cultivars/tissues/treatment in the Tomexpress database. Subsequently, global gene coexpression (GCN) analysis was carried out by calculating pairwise Pearson correlation coefficients between each PIF gene against the tomato genome was computed, and Fisher's Z-transformation was used to test the statistical significance of the pairwise correlations. 


\section{Quantification of metabolites}

Carotenoids, chlorophylls, tocopherols were extracted as described (Barja et al., 2021). Ethylene production in tomato fruit was assessed by adapting a previous method (Pereira et al., 2017). Specifically, we collected tomato fruits at MG stage and incubated them in long day greenhouse conditions in opened $50-\mathrm{mL}$ tubes. Once a day, the tubes were sealed with Parafilm (BEMIS) and incubated for one hour. After that hour, $30 \mathrm{~mL}$ of the headspace air was collected by injecting a syringe through the Parafilm layer. That samples were finally collected in a GC-MS tube and analyzed. GC-MS analysis was performed with an Agilent 7890A gas chromatograph coupled to a 5975C mass selective detector.

\section{Confocal and electron microscopy}

Subcellular localization, protein stability and BiFC experiments were determined by analyzing agroinfiltrated leaf samples with an Olympus FV 1000 confocal laser-scanning microscope. GFP signal and chlorophyll autofluorescence were detected using an argon laser for excitation (at $488 \mathrm{~nm}$ ) and a 500-510 nm filter for detection of GFP fluorescence and a $610-700 \mathrm{~nm}$ filter for detection of chlorophyll fluorescence. In the case of protein stability, same conditions were used strictly to take all the images. First, a field of $20-30$ nuclei was photographed. After that, the sample was incubated during $30 \mathrm{~min}$ in Red (200 $\mu \mathrm{mol} \mathrm{m}-2 \mathrm{~s}-1)$ and Far Red light $(100 \mu \mathrm{mol} \mathrm{m}-2 \mathrm{~s}-1)$. The exact same field was photographed again after that exposure, using the same parameters as before. The intensity of the fluorescence signal was measures using the ImageJ software. Individual Regions Of Interest (ROI) were created for each nucleus and identical-size ROI were used to compare the same nucleus before and after the light exposure. The intensity of fluorescence was measured in each pixel of all the ROI and each ROI was considered an individual replicate within one population.

Root samples were washed 2 washes on $10 \mathrm{~min}$ in absolute ethanol and after that they were dry by critical point using an equipment Emitech K850. Then, the samples were mounted on the supports of the SEM suing a biadhesive conductive disk and coated with a thin layer of gold (about $60 \mathrm{~nm}$ ) to make them conductive electrical. This was done with the SEM coating system model SC510 (Fisons Instruments). The study was carried out using a Field Emission Scanning Electron Microscope J-7001F (Jeol) using a secondary electron detector to analyze the topology of the samples. 


\section{REFERENCES}

Abdullah, E., Idris, A., and Saparon, A. (2017). TomExpress, a unified tomato RNA-Seq platform for visualization of expression data, clustering and correlation networks. ARPN J. Eng. Appl. Sci. 12: 3218-3221.

Ahrazem, O., Argandoña, J., Fiore, A., Aguado, C., Luján, R., Rubio-mora, Á., Marro, M., Andrade, C.A., and Loza-alvarez, P., Diretto, G., and Gómez-Gómez, L. (2018). Transcriptome analysis in tissue sectors with contrasting crocins accumulation provides novel insights into apocarotenoid biosynthesis and regulation during chromoplast biogenesis. Sci. Rep. 8: 2843.

Al-sady, B., Ni, W., Kircher, S., Schäfer, E., and Quail, P.H. (2006). Photoactivated phytochrome induces rapid PIF3 phosphorylation prior to proteasome-mediated degradation. Mol. Cell 23: 439-446.

Appenroth, K.J., Lenk, G., Goldau, L., and Sharma, R. (2006). Tomato seed germination: Regulation of different response modes by phytochrome B2 and phytochrome A. Plant Cell Environ. 29: 701-709.

Auge, G.A., Perelman, S., Crocco, C.D., Sánchez, R.A., and Botto, J.F. (2009). Gene expression analysis of light-modulated germination in tomato seeds. New Phytol. 183: 301314.

Barja MV, Ezquerro M, Beretta S, Diretto G, Florez-Sarasa I, Feixes E, Fiore A, Karlova R, Fernie AR, Beekwilder J, and Rodríguez-Concepción M. (2021) Several geranylgeranyl diphosphate synthase isoforms supply metabolic substrates for carotenoid biosynthesis in tomato. New Phytol. 231: 255-272

Belda-Palazon, B., Ruiz, L., Marti, E., Tarraga, S., Tiburcio, A.F., Culiañez, F., Farreas, R., Carrasco, P., and Ferrando, A. (2012). Aminopropyltransferases involved in polyamine biosynthesis localize preferentially in the nucleus of plant cells. PLoS One 7: e46907

Bianchetti, R.E., Freschi, L., Cruz, A.B., Alves, F.R.R., Rossi, M., Purgatto, E., and Peres, L.E.P. (2018). Light, ethylene and auxin signaling interaction regulates carotenoid biosynthesis during tomato fruit ripening. Front. Plant Sci. 9: 1-16.

Bou-Torrent, J., Toledo-Ortiz, G., Ortiz-Alcaide, M., Cifuentes-Esquivel, N., Halliday, K.J., Martinez-Garcia, J.F., and Rodriguez-Concepcion, M. (2015). Regulation of carotenoid biosynthesis by shade relies on specific subsets of antagonistic transcription factors and cofactors. Plant Physiol. 169: 1584-1594.

Bu, Q., Castillon, A., Chen, F., Zhu, L., and Huq, E. (2011a). Dimerization and blue light regulation of PIF1 interacting bHLH proteins in Arabidopsis. Plant Mol. Biol. 77: 501-511.

Bu, Q., Zhu, L., and Huq, E. (2011b). Multiple kinases promote light-induced degradation of PIF1. Plant Signal. Behav. 6: 1119-1121.

Casal, J.J., Luccioni, L.G., Oliverio, K.A., Boccalandro, H.E., and Casal, J.J. (2003). Light , phytochrome signalling and photomorphogenesis in Arabidopsis. Photochem. Photobiol. Sci. 2: 625-636.

Calvert, A. (1959). Effect of the Early Environment on the Development of Flowering in Tomato II. Light and Temperature Interactions. J. Hortic. Sci. 34: 154-162.

Camps, C. and Gilli, C. (2017). Prediction of local and global tomato texture and quality by FT-NIR spectroscopy and chemometric. Eur. J. Hortic. Sci. 82: 126-133.

Chenge-Espinosa, M., Cordoba, E., Romero-Guido, C., Toledo-Ortiz, G., and Leon, P. (2018). Shedding light on the methylerythritol phosphate (MEP)-pathway: long hypocothyl 5 (HY5)/ phytochrome-interacting factors (PIFs) transcription factors modulating key limiting steps. Plant J. 52: 1-14. 
bioRxiv preprint doi: https://doi.org/10.1101/2021.10.29.466498; this version posted November 1,2021 . The copyright holder for this preprint (which was not certified by peer review) is the author/funder, who has granted bioRxiv a license to display the preprint in perpetuity. It is made available under aCC-BY-NC-ND 4.0 International license.

Choi, H. and Oh, E. (2016). PIF4 Integrates Multiple Environmental and Hormonal Signals for Plant Growth Regulation in Arabidopsis. Mol. Cells 39: 587-593.

Ditta, G., Pinyopich, A., Robles, P., Pelaz, S., and Yanofsky, M.F. (2004). The SEP4 gene of Arabidopsis thaliana functions in floral organ and meristem identity. Curr. Biol. 14: 19351940.

Dielen, V., Marc, D., and Kinet, J.M. (1998). Flowering in the uniflora mutant of tomato (Lycopersicon esculentum Mill.): Description of the reproductive structure and manipulation of flowering time. Plant Growth Regul. 25: 149-157.

Fernandez, A.l. et al. (2009). Flexible tools for gene expression and silencing in tomato. Plant Physiol. 151: 1729-1740.

Fujisawa, M., Nakano, T., and Ito, Y. (2011). Identification of potential target genes for the tomato fruit-ripening regulator RIN by chromatin immunoprecipitation. BMC Plant Biol. 11: 26.

Gapper, N.E., McQuinn, R.P., and Giovannoni, J. (2013). Molecular and genetic regulation of fruit ripening. Plant Mol. Biol. 82: 575-591.

Giliberto, L., Perrotta, G., Pallara, P., Weller, J.L., Fraser, P.D., Bramley, P.M., Fiore, A., Tavazza, M., and Giuliano, G. (2005). Manipulation of the blue light photoreceptor cryptochrome 2 in tomato affects vegetative development, flowering time, and fruit antioxidant content. Plant Physiol. 137: 199-208.

Giménez, E., Pineda, B., Capel, J., Antón, M.T., Atarés, A., Pérez-Martín, F., García-Sogo, B., Angosto, T., Moreno, V., and Lozano, R. (2010). Functional analysis of the Arlequin mutant corroborates the essential role of the ARLEQUIN/TAGL1 gene during reproductive development of tomato. PLoS One 5: e14427.

Giovannoni, J. (2004). Genetic regulation of fruit development and ripening. Plant Cell 16: 170-181.

Gramegna, G., Rosado, D., S.nchez Carranza, A.P., Cruz, A.B., Sim.n-Moya, M., Llorente, B., Rodriguez-Concepcion, M., Freschi, L., and Rossi, M. (2018). PHYTOCHROMEINTERACTING FACTOR 3 mediates light-dependent induction of tocopherol biosynthesis during tomato fruit ripening. Plant Cell Environ. 42: 1328- 1339.

Huq, E., Al-Sady, B., Hudson, M., Kim, C., Apel, K., and Quail, P.H. (2004). Pytocrome interacting factor 1 is a critical bHLH regulator of chloropyll biosynthesis. Science 305: 19371941.

Itkin, M., Seybold, H., Breitel, D., Rogachev, I., Meir, S., and Aharoni, A. (2009). TOMATO AGAMOUS-LIKE 1 is a component of the fruit ripening regulatory network. Plant J. 60: 1081-1095.

Kabas, O. and Ozmerzi, A. (2008). Determining the mechanical properties of cherry tomato varieties for handling. J. Texture Stud. 39: 199-209.

Koini, M.A., Alvey, L., Allen, T., Tilley, C.A., Harberd, N.P., Whitelam, G.C., Franklin, K.A., and Le, L. (2009). High Temperature-Mediated Adaptations in Plant Architecture Require the bHLH Transcription Factor PIF4. Curr. Biol. 19: 408-413.

Kumar, I., Swaminathan, K., Hudson, K., and Hudson, M.E. (2016). Evolutionary divergence of phytochrome protein function in Zea mays PIF3 signaling. J. Exp. Bot. 67: 4231-4240.

Lau, O.S. and Deng, X.W. (2010). Plant hormone signaling lightens up: integrators of light and hormones. Curr. Opin. Plant Biol. 13: 571-577.

Lee, K.P., Piskurewicz, U., Turečková, V., Carat, S., Chappuis, R., Strnad, M., Fankhauser, C., and Lopez-Molina, L. (2012). Spatially and genetically distinct control of seed germination by phytochromes A and B. Genes Dev. 26: 1984-1996. 
bioRxiv preprint doi: https://doi.org/10.1101/2021.10.29.466498; this version posted November 1,2021 . The copyright holder for this preprint (which was not certified by peer review) is the author/funder, who has granted bioRxiv a license to display the preprint in perpetuity. It is made available under aCC-BY-NC-ND 4.0 International license.

Leivar, P. and Quail, P.H. (2011). PIFs: Pivotal components in a cellular signaling hub. Trends Plant Sci. 16: 19-28.

Liljegren, S.J., Ditta, G.S., Eshed, Y., Savidge, B., Bowman, J.L., and Yanofsky, M.F. (2000). SHATTERPROOF MADS-box genes control seed dispersal in Arabidopsis. Nature 404: 766-770.

Llorente, B., D'Andrea, L., and Rodriguez-Concepcion, M. (2016a). Evolutionary recycling of light signaling components in fleshy fruits: new insights on the role of pigments to monitor ripening. Front. Plant Sci. 7: 263.

Llorente, B., D’Andrea, L., Ruiz-Sola, M..., Botterweg, E., Pulido, P., Andilla, J., LozaAlvarez, P., and Rodriguez-Concepcion, M. (2016b). Tomato fruit carotenoid biosynthesis is adjusted to actual ripening progression by a light-dependent mechanism. Plant J. 85: 107119.

de Lucas, M. and Prat, S. (2014). PIFs get BRright: PHYTOCHROME INTERACTING FACTORs as integrators of light and hormonal signals. New Phytol. 202: 1126-1141

Lupi, A.C.D., Lira, B.S., Gramegna, G., Trench, B., Alves, F.R.R., Demarco, D., Peres, L.E.P., Purgatto, E., Freschi, L., and Rossi, M. (2019). Solanum lycopersicum GOLDEN 2-

LIKE 2 transcription factor affects fruit quality in a light- and auxin-dependent manner. PLoS One 14: e0212224.

Mancinelli, A.L., Borthwick, H.A., and Hendricks, S.B. (1966). Phytochrome action in tomatoseed germination. Bot. Gaz. 127: 1-5.

Monte, E., Tepperman, J.M., Al-Sady, B., Kaczorowski, K.A., Alonso, J.M., Ecker, J.R., Li, X., Zhang, Y., and Quail, P.H. (2004). The phytochrome-interacting transcription factor, PIF3, acts early, selectively, and positively in light-induced chloroplast development. Proc. Natl. Acad. Sci. U. S. A. 101: 16091-16098.

Moon, J., Zhu, L., Shen, H., and Huq, E. (2008). PIF1 directly and indirectly regulates chlorophyll biosynthesis to optimize the greening process in Arabidopsis. Proc. Natl. Acad. Sci. U. S. A. 105: 9433-9438.

Ni, M., Tepperman, J.M., and Quail, P.H. (1998). PIF3, a phytochrome-interacting factor necessary for normal photoinduced signal transduction, is a novel basic helix-loop-helix protein. Cell 95: 657-667.

Oh, E., Kang, H., Yamaguchi, S., Park, J., Lee, D., Kamiya, Y., and Choi, G. (2009). Genome-wide analysis of genes targeted by PHYTOCHROME INTERACTING FACTOR 3LIKE5 during seed germination in Arabidopsis. Plant Cell 21: 403-419.

Oh, E., Kim, J., Park, E., Kim, J.-I., Kang, C., and Choi, G. (2004). PIL5, a phytochrome interacting basic helix-loop-helix protein, is a key negative regulator of seed germination in Arabidopsis thaliana. Plant Cell 16: 3045-3058.

Oh, E., Yamaguchi, S., Hu, J., Yusuke, J., Jung, B., Paik, I., Lee, H.-S., Sun, T. -p., Kamiya, Y., and Choi, G. (2007). PIL5, a phytochrome-interacting bHLH Protein, regulates gibberellin responsiveness by binding directly to the GAI and RGA Promoters in Arabidopsis seeds.

Plant Cell 19: 1192-1208.

Oh, E., Yamaguchi, S., Kamiya, Y., Bae, G., Chung, W. II, and Choi, G. (2006). Light activates the degradation of PIL5 protein to promote seed germination through gibberellin in Arabidopsis. Plant J. 47: 124-139.

Park, E., Kim, Y., and Choi, G. (2018). Phytochrome B requires PIF degradation and sequestration to induce light responses across a wide range of light conditions. Plant Cell 30: 1277-1292.

Park, E., Park, J., Kim, J., Nagatani, A., Lagarias, J.C., and Choi, G. (2012). Phytochrome B inhibits binding of phytochrome-interacting factors to their target promoters. Plant J. 72: 537546. 
Paik, I., Kathare, P.K., Kim, J. II, and Huq, E. (2017). Expanding roles of PIFs in signal integration from multiple processes. Mol. Plant 10: 1035-1046.

Pedmale, U. V, Huang, S.C., Zander, M., Cole, B.J., Hetzel, J., Ljung, K., Reis, P.A.B., Sridevi, P., Nito, K., Nery, J.R., Ecker, J.R., and Chory, J. (2016). Cryptochromes interact directly with PIFs to control plant growth in limiting blue light. Cell 164: 233-245.

Pesaresi, P., Mizzotti, C., Colombo, M., and Masiero, S. (2014). Genetic regulation and structural changes during tomato fruit development and ripening. Front. Plant Sci. 5: 124.

Pham, V.N., Kathare, P.K., and Huq, E. (2018). Phytochromes and Phytochrome Interacting Factors. Plant Physiol. 176: 1025-1038.

Qiu, Y., Pasoreck, E.K., Reddy, A.K., Nagatani, A., Ma, W., Chory, J., and Chen, M. (2017). Mechanism of early light signaling by the carboxy-terminal output module of Arabidopsis phytochrome B. Nat. Commun. 8: 1905.

Pereira, L., Pujol, M., Garcia-Mas, J., and Phillips, M.A. (2017). Non-invasive quantification of ethylene in attached fruit headspace at 1 p.p.b. by gas chromatography-mass spectrometry. Plant J. 91: 172-183.

Reed, J.W., Nagpal, P., Pooe, D.S., Furuya, M., and Chorya, J. (1993). Mutations in the Gene for the Red / Far-Red Light Receptor Phytochrome B Alter Cell Elongation and Physiological Responses throughout Arabidopsis Development. 5: 147-157.

Ritter, A. et al. (2017). The transcriptional repressor complex FRS7-FRS12 regulates flowering time and growth in Arabidopsis. Nat. Commun. 8: 15235.

Rodriguez-Concepcion, M. et al. (2018). A global perspective on carotenoids: Metabolism, biotechnology, and benefits for nutrition and health. Prog. Lipid Res. 70: 62-93.

Rosado, D., Gramegna, G., Cruz, A., Lira, B.S., Freschi, L., De Setta, N., and Rossi, M. (2016). Phytochrome Interacting Factors (PIFs) in Solanum lycopersicum: Diversity, evolutionary history and expression profiling during different developmental processes. PLoS One 11: e0165929.

Rosado, D., Trench, B., Bianchetti, R.E., Zuccarelli, R., Alves, F.R.R., Purgatto, E., Floh, E.I.S., Nogueira, F.T., Freschi, L., and Rossi, M. (2019). Downregulation of PHYTOCHROME-INTERACTING FACTOR 4 influences plant development and fruit production. Plant Physiol. 181: 1360-1370.

Sakuraba, Y., Jeong, J., Kang, M.-Y.Y., Kim, J., Paek, N.-C.C., and Choi, G. (2014). Phytochrome-interacting transcription factors PIF4 and PIF5 induce leaf senescence in Arabidopsis. Nat. Commun. 5: 4636.

Sato, S. et al. (2012). The tomato genome sequence provides insights into fleshy fruit evolution. Nature 485: 635-641.

Seo, M., Nambara, E., Choi, G., and Yamaguchi, S. (2009). Interaction of light and hormone signals in germinating seeds. Plant Mol. Biol. 69: 463-472.

Seo, H.S., Watanabe, E., Tokutomi, S., Nagatani, A., and Chua, N. (2004). Photoreceptor ubiquitination by COP1 E3 ligase desensitizes phytochrome A signaling. Genes Dev. 18: 617-622.

Seymour, G.B., Østergaard, L., Chapman, N.H., Knapp, S., and Martin, C. (2013). Fruit Development and Ripening. Annu. Rev. Plant Biol. 64: 219-241.

Shinomura, T., Nagatani, A., Chory, J., and Furuya, M. (1994). The induction of seed germination in Arabidopsis thaliana is regulated principally by phytochrome $B$ and secondarily by phytochrome A. Plant Physiol. 104: 363-371.

Song, Y., Yang, C., Gao, S., Zhang, W., Li, L., and Kuai, B. (2014). Age-Triggered and DarkInduced Leaf Senescence Require the bHLH Transcription Factors PIF3, 4, and 5. Mol. Plant 7: 1776-1787. 
Shen, H., Moon, J., and Huq, E. (2005). PIF1 is regulated by light-mediated degradation through the ubiquitin-26S proteasome pathway to optimize photomorphogenesis of seedlings in Arabidopsis. Plant J. 44: 1023-1035.

Shen, H., Zhu, L., Castillon, A., Majee, M., Downie, B., and Huq, E. (2008). Light-Induced Phosphorylation and Degradation of the Negative Regulator PHYTOCHROME-

INTERACTING FACTOR1 from Arabidopsis Depend upon Its Direct Physical Interactions with Photoactivated Phytochromes. Plant Cell 20: 1586-1602.

Shi, H., Zhong, S., Mo, X., Liu, N., Nezames, C.D., and Deng, X.W. (2013). HFR1

Sequesters PIF1 to Govern the Transcriptional Network Underlying Light-Initiated Seed Germination in Arabidopsis. Plant Cell 25: 3770-3784.

Shichijo, C., Katada, K., Tanaka, O., and Hashimoto, T. (2001). Phytochrome A-mediated inhibition of seed germination in tomato. Planta 213: 764-769.

Soyk, S., Müller, N.A., Park, S.J., Schmalenbach, I., Jiang, K., Hayama, R., Zhang, L., Van Eck, J., Jiménez-Gómez, J.M., and Lippman, Z.B. (2017). Variation in the flowering gene SELF PRUNING 5G promotes day-neutrality and early yield in tomato. Nat. Genet. 49: 162168.

Su, L., Hou, P., Song, M., Zheng, X., Guo, L., Xiao, Y., and Yan, L. (2015). Synergistic and Antagonistic Action of Phytochrome (Phy) A and PhyB during Seedling De-Etiolation in Arabidopsis thaliana. Int. J. Mol. Sci. 16: 12199-12212.

Toledo-ortiz, G., Huq, E., and Quail, P.H. (2003). The Arabidopsis Basic / Helix-Loop-Helix Transcription Factor Family. 15: 1749-1770.

Toledo-Ortiz, G., Huq, E., and Rodriguez-Concepcion, M. (2010). Direct regulation of phytoene synthase gene expression and carotenoid biosynthesis by phytochrome interacting factors. Proc. Natl. Acad. Sci. 107: 11626-11631.

The HY5-PIF regulatory module coordinates light and temperature control of photosynthetic gene transcription.

Toledo-Ortiz, G., Johansson, H., Lee, K.P., Bou-Torrent, J., Stewart, K., Steel, G., Rodríguez-Concepción, M., and Halliday, K.J. (2014) PLoS Genet. 10: e1004416.

Thines, B.C., Youn, Y., Duarte, M.I., and Harmon, F.G. (2014). The time of day effects of warm temperature on flowering time involve PIF4 and PIF5. J. Exp. Bot. 65: 1141-1151.

Trupkin, S.A., Legris, M., Buchovsky, A.S., Belén, M., Rivero, T., and Casal, J.J. (2014). Phytochrome $B$ nuclear bodies respond to the low red to far-red ratio and to the reduced irradiance of canopy. Plant Physiol. 165: 1698-1708.

Vrebalov, J., Pan, I.L., Arroyo, A.J.M., McQuinn, R., Chung, M., Poole, M., Rose, J., Seymour, G., Grandillo, S., Giovannoni, J., and Irish, V.F. (2009). Fleshy fruit expansion and ripening are regulated by the tomato SHATTERPROOF gene TAGL1. Plant Cell 21: 30413062.

Wang, C., Meng, L., Gao, Y., Grierson, D., and Fu, D. (2018). Manipulation of light signal transduction factors as a means of modifying steroidal glycoalkaloids accumulation in tomato leaves. Front. Plant Sci. 9: 437.

Wu, M., Liu, D., Abdul, W., Upreti, S., Liu, Y., Song, G., Wu, J., Liu, B., and Gan, Y. (2018). PIL5 represses floral transition in Arabidopsis under long day conditions. Biochem. Biophys. Res. Commun. 499: 513-518.

Zhang, Y., Liu, Z., Chen, Y., He, J.X., and Bi, Y. (2015). PHYTOCHROME-INTERACTING FACTOR 5 (PIF5) positively regulates dark-induced senescence and chlorophyll degradation in Arabidopsis. Plant Sci. 237: 57-68. 
bioRxiv preprint doi: https:/doi.org/10.1101/2021.10 29.466498- this version posted November 1 2021. The copyright holder for this preprint (which was not certified by peer review) is the author/funder, who has granted bioRxiv a license to display the preprint in perpetuity. It is made available under aCC-BY-NC-ND 4.0 International license.

\section{FIGURES}

A

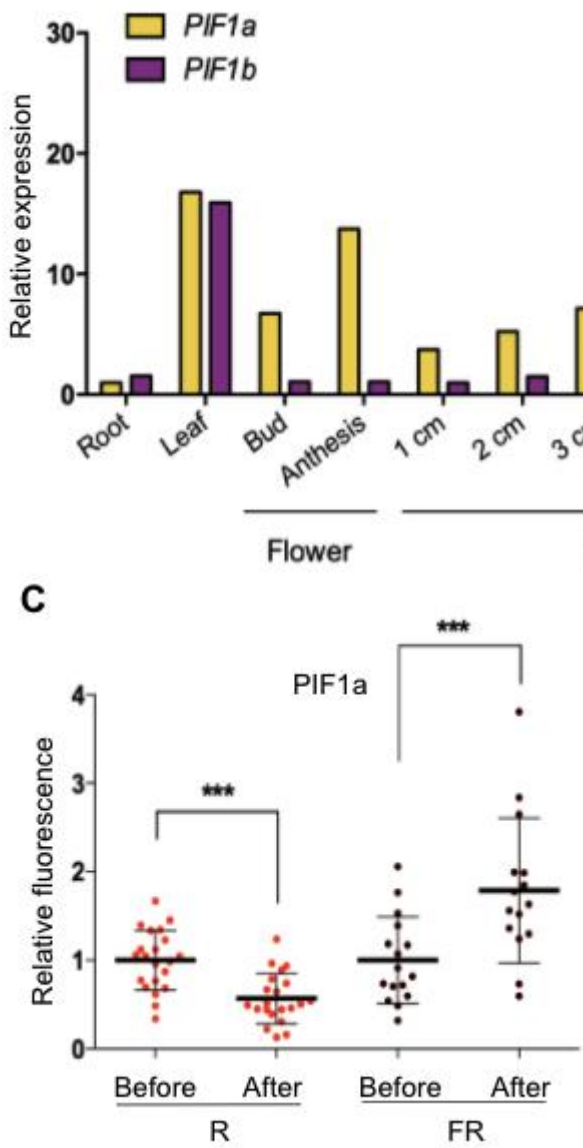

D
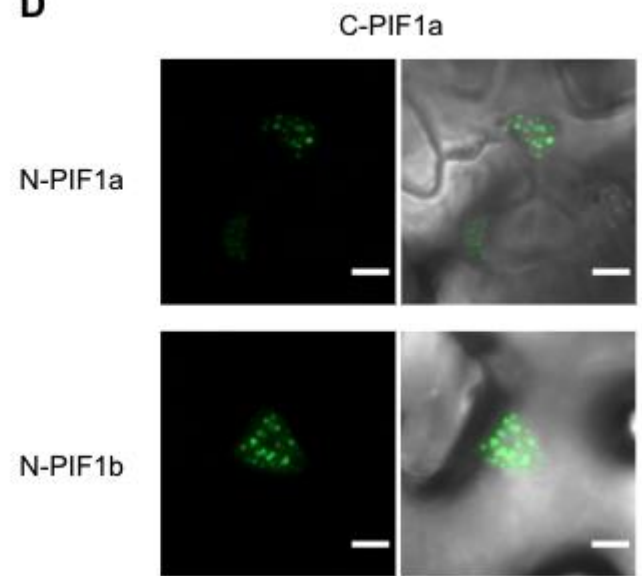

B

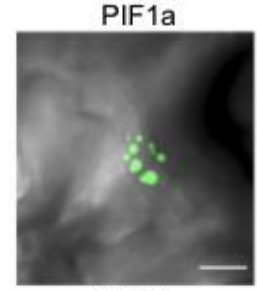

PIF1b

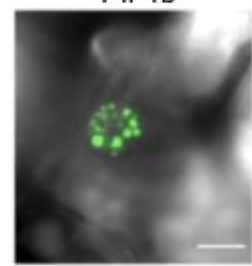

Fruit
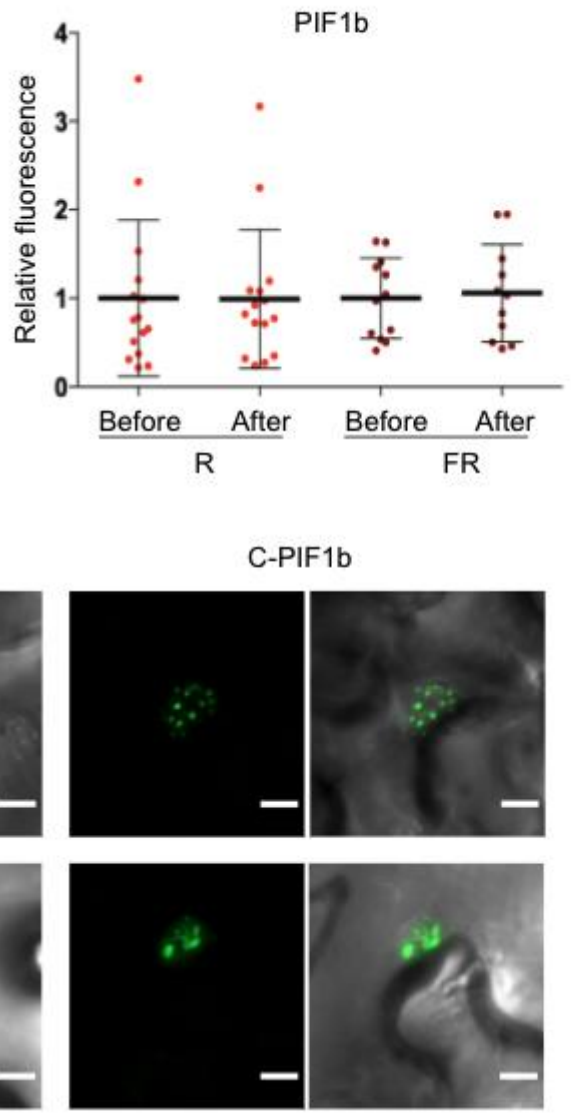
Fig 1. Expression profiles and protein features of PIF1a and PIF1b.

A. Levels of PIF1a and PIF1b transcripts in different plant organs. Data are obtained from BAR (University of Toronto) and expressed relative to the lowest expression level found in the study (i.e. PIF1a expression in roots).

B. Confocal microscopy images of GFP fluorescence in the nucleus of $\mathrm{N}$. benthamiana leaf cell transiently expressing PIF1a or PIF1b fused to GFP. Scale bar $=10 \mu \mathrm{m}$

C. Quantification of GFP fluorescence of nuclei from leaf areas. Error bars indicate SD of at least 15 different nuclei. Asterisks mark statistically significant changes in student's test $(* * *=p<0.001)$

D. BiFC analysis of PIF1a and PIF1b protein-protein interaction.Confocal microscopy images of GFP fluorescence in $\mathrm{N}$. benthamiana leaf cells transiently expressing the indicated proteins fused to $\mathrm{N}$ - or $\mathrm{C}$-terminal GFP halves for $\mathrm{BiFC}$ analysis. Images of representative nuclei showing either GFP fluorescence alone (left) or overlapped with bright field images (right) are shown for every combination. Scale bar $=10 \mu \mathrm{m}$. 


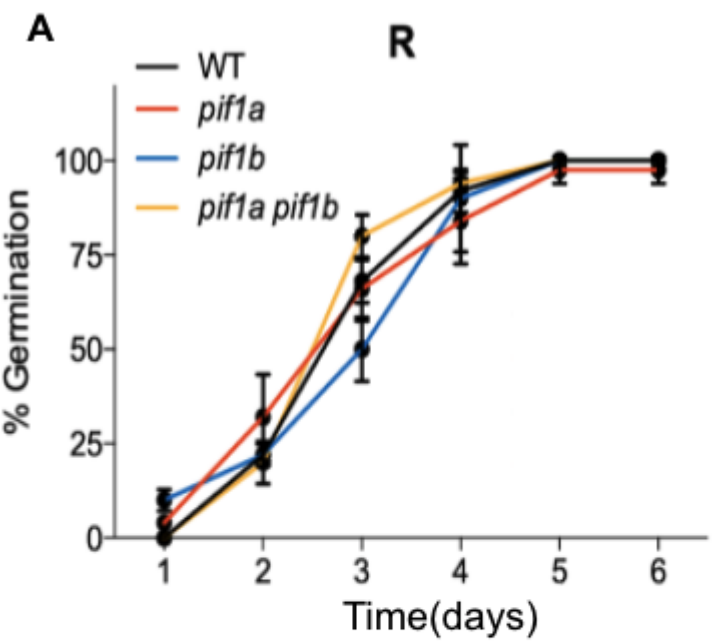

FR

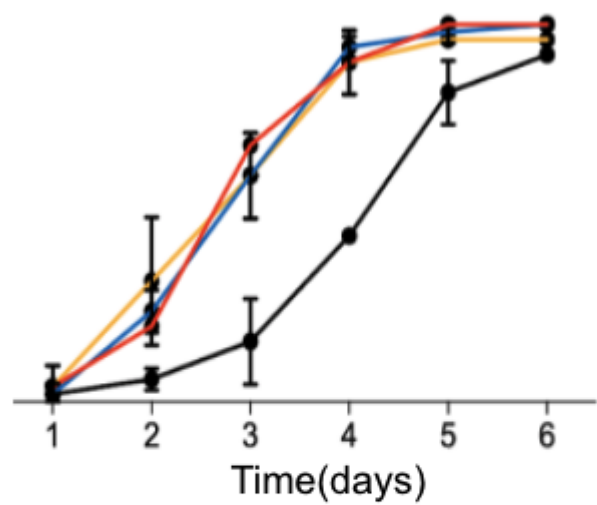

B

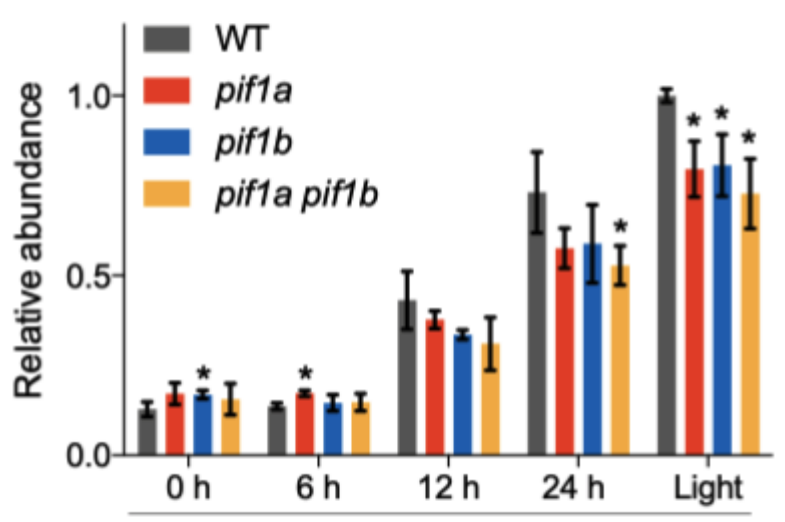

Carotenoids

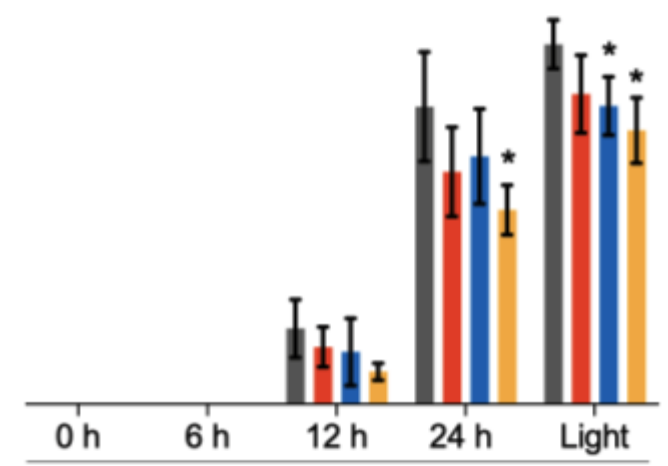

Chlorophylls

Fig 2. Germination and de-etiolation assays.

A. Kinetics of germination of the 4 indicated genotypes under hourly pulses of $\mathrm{R}$ (left) or FR (right) for 6 days. Error bars indicate SD of 2 biological replicates with at least 50 seeds each.

B. Carotenoid and chlorophyll levels during de-etiolation. WT and mutant seedlings were germinated in the dark for 7 days and then illuminated with white light for the indicated times. Seedlings germinated and grown under continuous white light for 7 days were used as controls. Carotenoid and chlorophyll levels are represented relative to those in light-grown WT seedlings. Error bars indicate SD of at least 3 biological replicates. Asterisks mark statistically significant changes in the indicated genotype compared to the WT according to t-student test $\left({ }^{*}=p<0.05\right)$. 

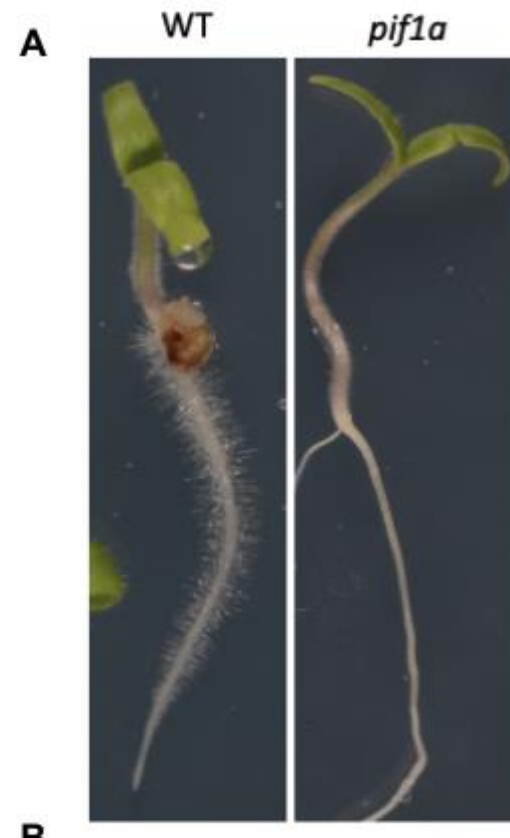

pif1b

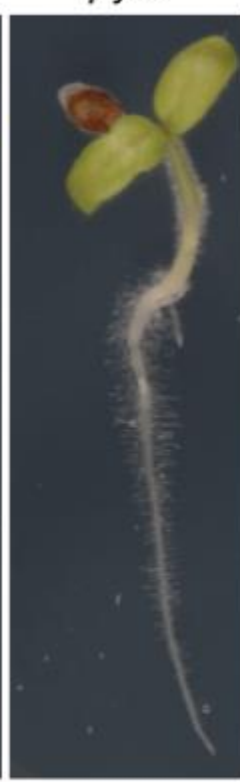

pif1a pif1b

B
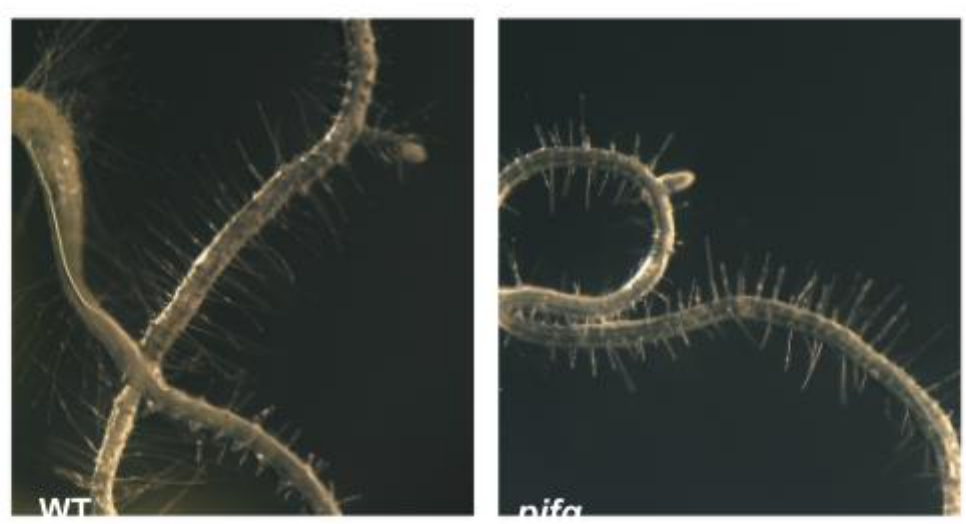

C
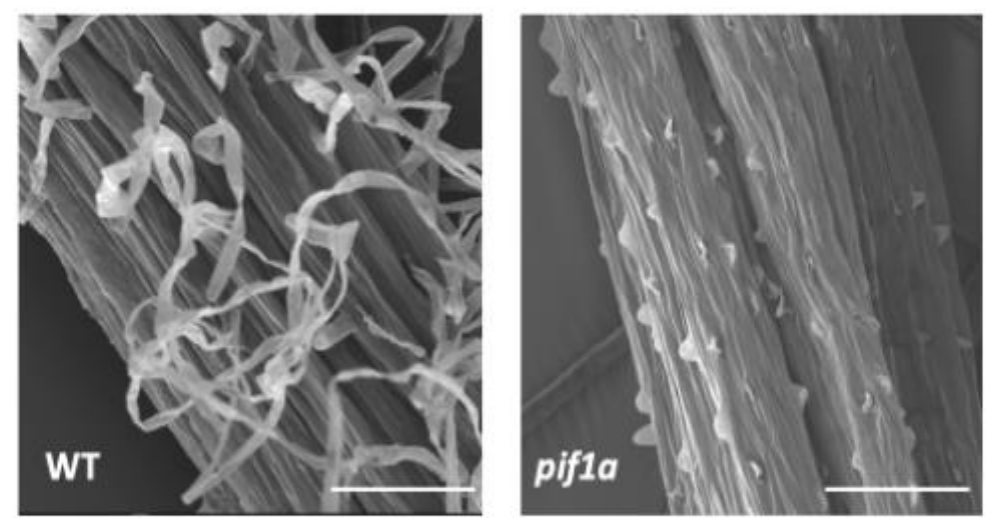

Fig 3. Root hair development in pif mutants.

A. Visual phenotype of WT and pif1 mutant seedlings.

B. Arabidopsis roots of WT and pifq mutant.

C. SEM images of tomato WT and pif1 mutant roots. Scale bar $=100 \mu \mathrm{m}$ 
A

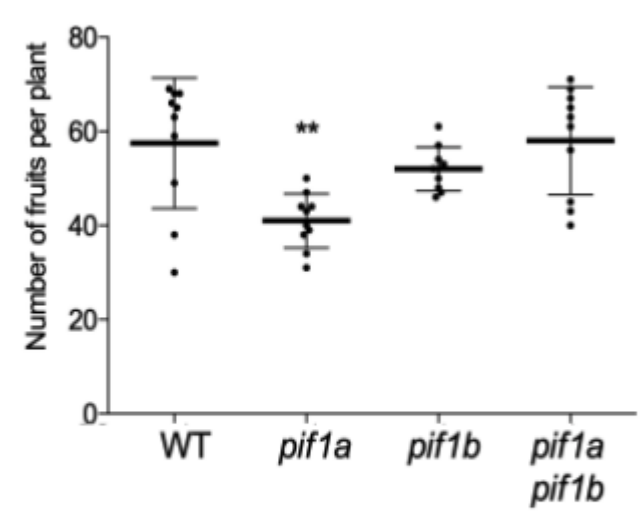

C

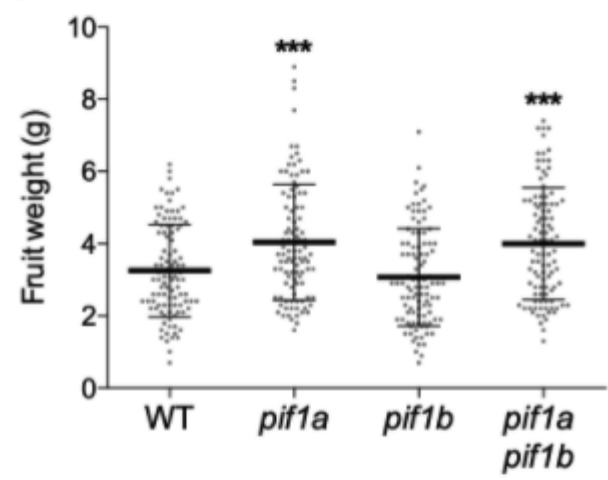

B

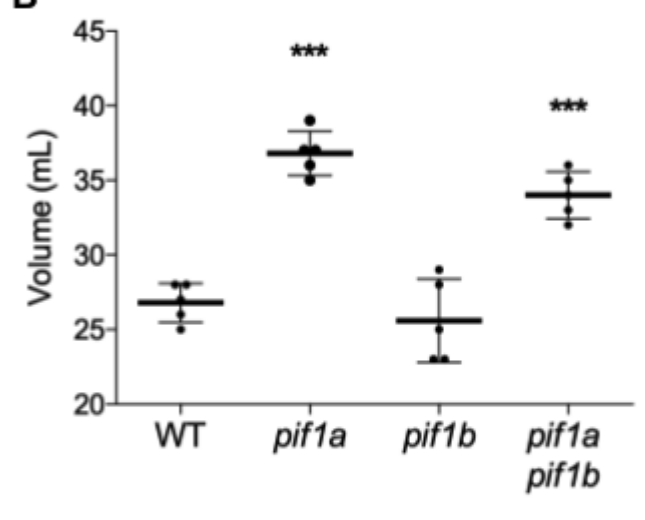

D

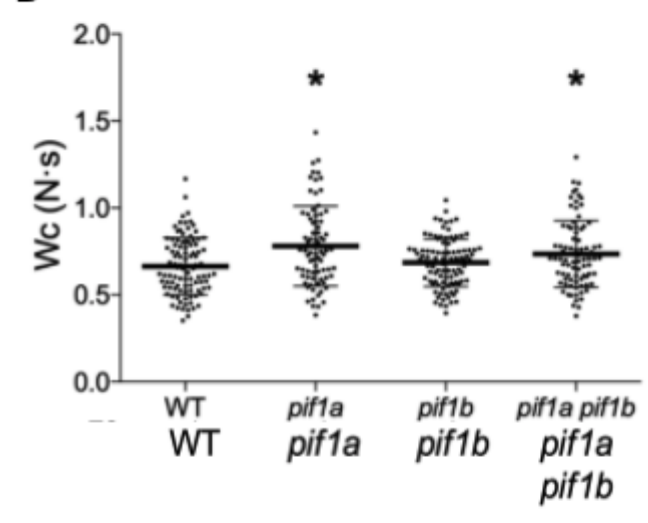

Fig 4. Fruit traits in pif1 mutants.

A. Fruit yield in tomato pif1 mutants. Plot represents the total number of fruits produced by individual 19-week-old plants grown on soil in the greenhouse under long day conditions.

B. Fruit volume in tomato pif1 mutants. Plot represents the volume of 5 groups of 10 fruits each. Fruits were collected at the RR stage from plants grown on soil in the greenhouse under long day conditions.

C. Weight of 100 individual RR fruits of each genotype. Error bars indicate SD.

D. Fruit texture in tomato pif1 mutants. Plot represents Wc measurements (compression tests) of 100 individual RR fruits of each genotype.

Error bars indicate SD. Asterisks mark statistically significant changes in the indicated genotype compared with WT according to one-way ANOVA ( ${ }^{*}=p<$ $0.05,{ }^{* *}=p<0.01,{ }^{* * *}=p<0.001$ ) 


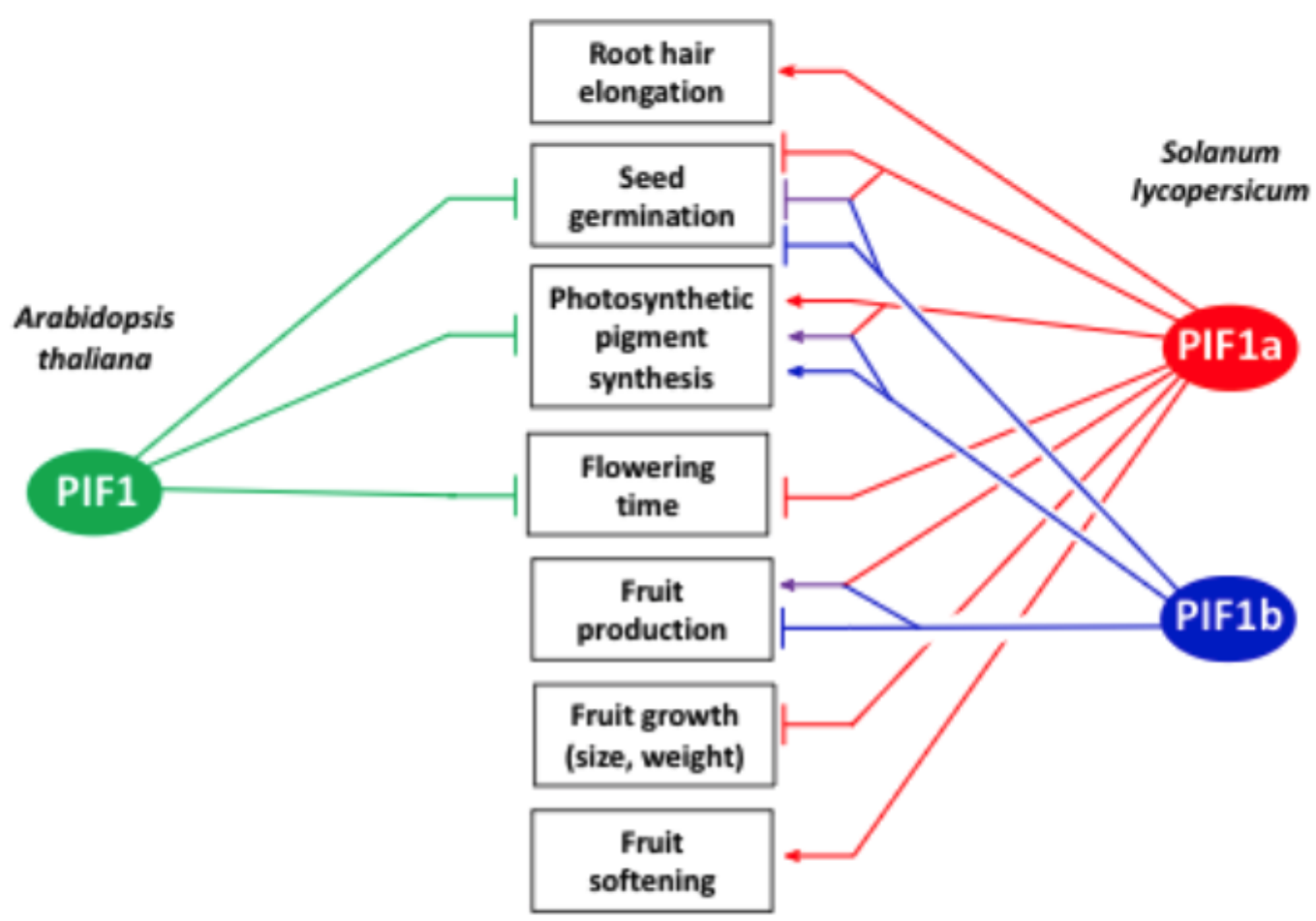

Fig 5. Schematic summary of proposed roles for PIF1 homologs in Arabidopsis and tomato. Arrows represent induction and bars represent repression. Purple lines represent functions of PIF1a-PIF1b heterodimers as deduced from this work. 